\title{
Mechanical properties of ZnO epitaxial layers grown on a- and $c$-axis sapphire
}

\author{
V. A. Coleman, ${ }^{\text {a) }}$ J. E. Bradby, and C. Jagadish \\ Department of Electronic Materials Engineering, Research School of Physical Sciences and Engineering, \\ The Australian National University, Canberra ACT 0200, Australia \\ P. Munroe \\ Electron Microscope Unit, University of New South Wales, Sydney NSW 2052, Australia
}

Y. W. Heo, S. J. Pearton, and D. P. Norton

Materials Science and Engineering, University of Florida, Gainesville, Florida 32611

\author{
M. Inoue and M. Yano \\ New Materials Research Centre, Osaka Institute of Technology, Osaka 535-8585, Japan
}

(Received 8 October 2004; accepted 5 April 2005; published online 11 May 2005)

\begin{abstract}
The mechanical properties of zinc oxide epitaxial layers grown on $a$ - and $c$-axis sapphire have been studied by spherical nanoindentation and cross-sectional transmission electron microscopy. As-grown threading dislocations, which are characteristic of epitaxial material, combined with the presence of the much harder, underlying substrate are found to have a significant effect on the mechanical behavior of $\mathrm{ZnO}$ epilayers as compared to bulk material. Epilayer material is found to be significantly harder than its bulk counterpart. For $a$-axis epilayers, analysis of load-unload data yields a hardness of $6.6 \pm 1.2 \mathrm{GPa}$, and $5.75 \pm 0.8 \mathrm{GPa}$ for $c$-axis layers. We attribute this increased hardness to strain compensation via the presence of as-grown defects. These defects inhibit the slip mechanism responsible for relative softness of bulk single crystals. The absence of pop-in events from analyzed continuous-load nanoindentation data is further evidence for strain compensation by native defects within the epilayers. Large variations in the spread of collected data are indicative of inhomegenity in the epilayers. C 2005 American Institute of Physics. [DOI: 10.1063/1.1929874]
\end{abstract}

With a wide band gap of $\sim 3.4 \mathrm{eV}$ and an exciton binding energy of $60 \mathrm{meV}$ at room temperature, $\mathrm{ZnO}$ is an attractive material for short-wavelength optoelectronic devices, such as blue light-emitting diodes and laser diodes. ${ }^{1}$ Several other characteristics further enhance the interest in this material for device manufacture, including good resistance to radiation damage, ${ }^{1}$ possibility for wet chemical etching, ${ }^{1}$ and low-power threshold for optical pumping. ${ }^{2}$ Recent advances in the growth of both high-quality $\mathrm{ZnO}$ single crystals ${ }^{1,2}$ and epitaxial layers ${ }^{1,3,4}$ have brought the realization of $\mathrm{ZnO}$ devices within reach. A key factor in device production is the way in which the active material is affected by surface contact. Semiconductor device processing involves extensive surface contact, and given that $\mathrm{ZnO}$ is a very soft ${ }^{5}$ material highly susceptible to damage, the properties that make it so attractive as an (opto)electronic device material may be compromised. Nanoindentation is a useful method for studying the mechanical properties of a material while also producing contact-induced damage. In particular, the highly localized nature of the indentation technique makes it ideal for simulating the material damage introduced during semiconductor processing.

Previously, nanoindentation studies of $\mathrm{ZnO}$ have focused on polycrystalline ${ }^{6,7}$ or single-crystal ${ }^{5,8,9}$ samples. A recent study reported that contact induces extensive deformation damage in $c$-axis $\mathrm{ZnO}$ single crystal samples, with a very large propagation of defects well beyond the volume under contact. ${ }^{8}$ This study showed that the production of these defects lead to quenching of excitonic luminescence, which,

\footnotetext{
${ }^{\text {a)} E l e c t r o n i c ~ m a i l: ~ v i c t o r i a . c o l e m a n @ a n u . e d u . a u ~}$
}

due to the extensive defect propagation, extended far from the indentation region. In the present study, we investigate the material properties and contact-induced damage on $a$ and $c$-axis epitaxial $\mathrm{ZnO}$ grown on sapphire. Epitaxial $\mathrm{ZnO}$ will be important for $\mathrm{ZnO}$ based devices, and thus a thorough understanding of the mechanical properties of this system is required. In the present study, we reveal that the mechanical properties of the epilayer material are strongly influenced by the presence of the underlying substrate, and also to the concentration of as-grown defects in the epimaterial. The large lattice mismatch ${ }^{4}$ between sapphire and $\mathrm{ZnO}$ results in dislocated epitaxial layers, with a typical dislocation density ${ }^{10}$ of $\sim 10^{7} \mathrm{~cm}^{-2}$. The sapphire substrate and high dislocation density also have an effect on the contact-induced damage in these samples. All of these factors combine to make the mechanical properties of epitaxial $\mathrm{ZnO}$ very different from that of bulk material, and has significant implications for device manufacture.

The high-quality $\mathrm{ZnO}$ epilayer samples used in this study were grown on $a-(1 \overline{1} 20)$ and $c$-(0001) axis sapphire, respectively. The $a$-axis layers were grown by molecular beam epitaxy (MBE), while the $c$-axis samples were grown by pulsed laser deposition. In both cases, the epilayer was $\sim 600 \mathrm{~nm}$ thick. Both the $a$ - and $c$-axis material had dislocation densities of $\sim 1 \times 10^{7} \pm 2.5 \times 10^{6} \mathrm{~cm}^{-2}$. The epitaxial material was compared with $c$ - and $a$-axis single crystal bulk material obtained from CERMET and MTI corporation, respectively. Series of both continuous load and partial loadunload nanoindentations were made with an Ultra-Micro Indentation System 2000 (UMIS) up to a maximum load of $50 \mathrm{mN}$. All measurements were carried out in ambient con- 

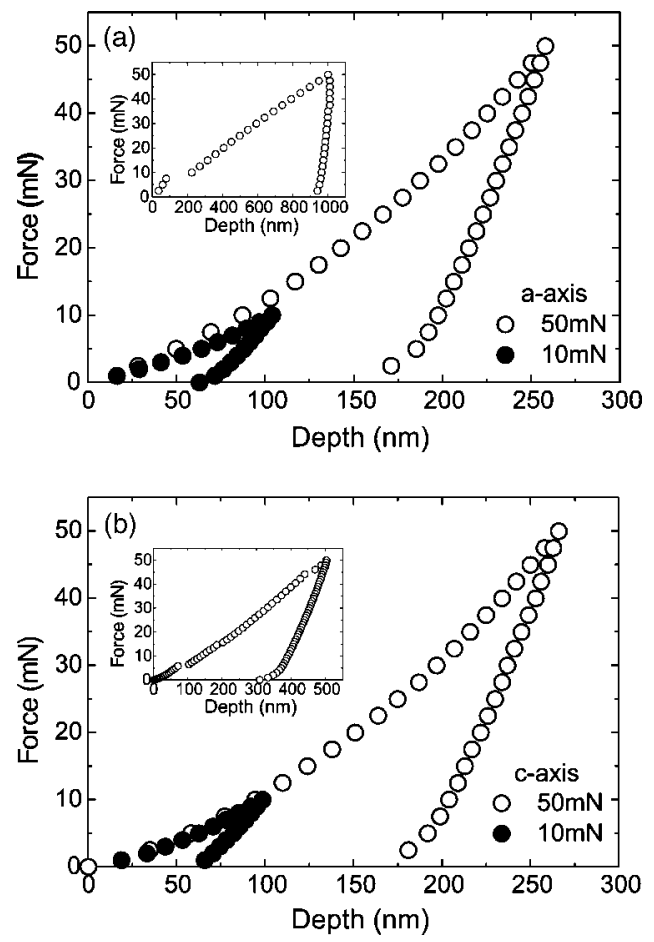

FIG. 1. Load-unload curves of (a) $a$-axis and (b) $c$-axis $\mathrm{ZnO}$ loaded to 10 and $50 \mathrm{mN}$. Insets show load-unload curves for single crystal bulk $\mathrm{ZnO}$ of corresponding orientation. It can be seen that no pop-in events are seen for the epitaxial $\mathrm{ZnO}$, whereas the bulk $\mathrm{ZnO}$ has either a single pop-in event ( $a$ axis) or multiple pop-in events ( $c$ axis).

ditions, with a spherical diamond indenter (radius $\sim 4.3 \mu \mathrm{m}$ ). All indents were made to less than $10 \%$ of the total film thickness to ensure that the mechanical properties of the sapphire did not influence those of the $\mathrm{ZnO} .{ }^{11}$ Both the UMIS and the indenter tip were precalibrated using a fused silica standard with known material properties. The Field and Swain method of analysis ${ }^{12}$ was used to extract hardness and elastic modulus values. Following indentation, the epilayers were prepared for cross-sectional transmission electron microscopy $(\mathrm{XTEM})$ analysis using an FEI $\times \mathrm{P} 200$ focused-ion beam (FIB) system with $30 \mathrm{keV} \mathrm{Ga}$ ions. Prior to milling, the FIB was used to deposit an $\sim 1 \mu \mathrm{m}$ thick Pt layer to protect the $\mathrm{ZnO}$ surface. XTEM images were taken on a Phillips CM 300 transmission electron microscope, using an accelerating voltage of $300 \mathrm{kV}$.

In Fig. 1, the continuous load-unload force-displacement curves are shown for both $a$ - and $c$-axis epilayers indented to 10 and $50 \mathrm{mN}$ [Figs. 1(a) and 1(b), respectively] and bulk material [insets] indented to $50 \mathrm{mN}$. The force-displacement curves for the epilayers are continuous, and no pop-in events are evident. This is very different from the behavior observed for the bulk material, which shows multiple pop-in events for the $c$-axis material. ${ }^{5}$ We have also recently investigated the mechanical properties of single-crystal bulk $a$-axis $\mathrm{ZnO}$, in which we observed a single but much larger pop-in discontinuity. These results will be reported elswhere. Pop-in events in single crystal samples can be attributed to slip along the basal and pyramidal planes, and is different for $a$ and $c$-axis samples due to the orientation of the basal plane in each case. ${ }^{8}$ The absence of pop-in events in the epitaxial material is indicative of some other strain compensation site present within the material. To investigate what this may be, we now turn to an XTEM analysis of the epitaxial material. Downloaded 25 Jul 2007 to 150.203 .178 .22 . Redistribution subject (a)

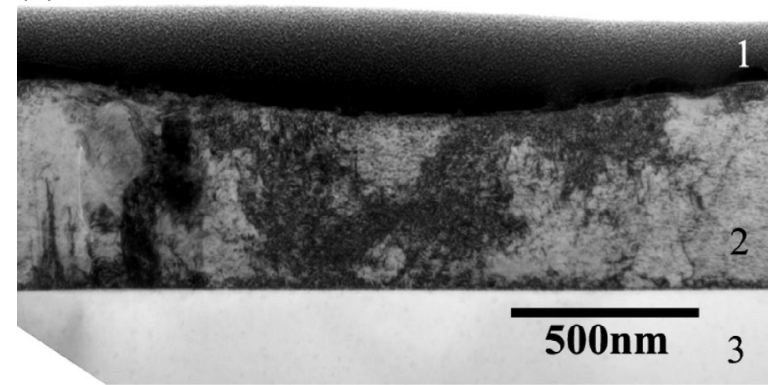

(b)

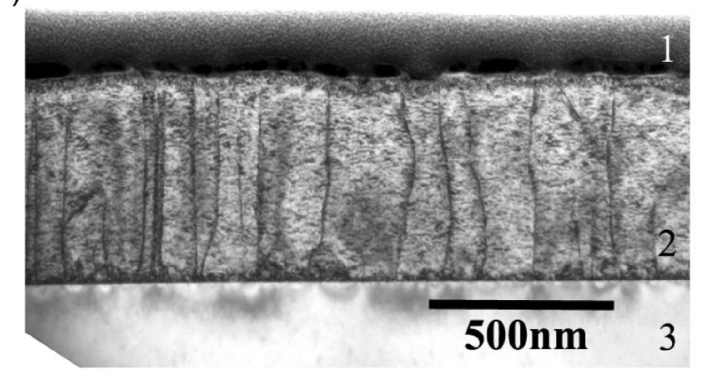

FIG. 2. (a) BF XTEM image of an indent in $a$-axis epi $\mathrm{ZnO}$. While some ion-beam induced damage to the $\mathrm{ZnO}$ is evident, there is no delamination of the $\mathrm{ZnO}$ film. The area directly under the indent shows considerable damage buildup, however no slip lines are evident. All indents were made using a continuous load cycle with an $\sim 4.3 \mu \mathrm{m}$ radius indenter to a maximum load of $50 \mathrm{mN}$. Region 1 is platinum deposited to protect the $\mathrm{ZnO}$ surface during FIB milling, 2 denotes the $\mathrm{ZnO}$, and region 3 is the underlying sapphire layer; (b) BF XTEM image of the $\mathrm{ZnO}$ taken away from the indent region, and shows a number of threading dislocations that are present throughout the material.

A bright-field XTEM image of a $50 \mathrm{mN}$ load indent in $a$-axis $\mathrm{ZnO}$ is shown in Fig. 2(a). While extensive deformation-induced damage is evident directly underneath the indent, there is no propagation of this damage into the sapphire substrate. There is also no evidence of cracking, or delamination of the $\mathrm{ZnO}$ film from the sapphire substrate, and, unlike the bulk material, there is no evidence of clear slip lines. Like the bulk material, ion beam damage to the $\mathrm{ZnO}$ induced during FIB milling is evident, giving the cross section a textured appearance. Selected area diffraction patterns taken directly underneath the indent within the region exposed to the greatest hydrostatic stress, show no evidence of a phase change. Another feature apparent from XTEM is threading dislocations within the epitaxial material [see inset of Fig. 2(b)]. These dislocations were present throughout both the $a$ - and $c$-axis material, and extended from the sapphire to the surface with evidence of their presence seen on the surface of the layers during FIB imaging. We propose that the presence of these dislocations inhibits slip by acting as strain compensation sites. Also, the very hard underlying sapphire substrate retards the propagation of defects in the plane perpendicular to the sample surface. Combined, both of these factors are responsible for the absence of pop-in events in the epitaxial layers.

We now turn to the analysis of hardness and elastic modulus data for the layers. The hardness and elastic modulus of the epilayers at a penetration below contact of $50 \mathrm{~nm}$ is shown in Table I and compared with data for the bulk material. From these data, it appears that the $a$-axis epilayers are harder than the $c$-axis layers. This is the opposite to what o AlP license or copyright, see http://apl.aip.org/apl//copyright.jsp 
TABLE I. Hardness and modulus as measured for $a$ - and $c$-axis $\mathrm{ZnO}$ epi and bulk layers at a penetration of $50 \mathrm{~nm}$ below contact.

\begin{tabular}{cccc}
\hline \hline Orientation & Sample type & $\begin{array}{c}\text { Hardness } \\
(\mathrm{GPa})\end{array}$ & $\begin{array}{c}\text { Modulus } \\
(\mathrm{GPa})\end{array}$ \\
\hline$a$ & epitaxial & $6.6 \pm 1.2$ & $318 \pm 50$ \\
$c$ & epitaxial & $5.75 \pm 0.8$ & $310 \pm 40$ \\
$a$ & bulk & $2 \pm 0.2$ & $163 \pm 6$ \\
$c$ & bulk & $4.8 \pm 0.2$ & $143 \pm 6$ \\
\hline \hline
\end{tabular}

has been observed in bulk material; however, the relative difference in hardness between the epilayers is small and may be attributed to differences in the material quality rather than as a result of the film orientation. Compared to the bulk material, the epilayers are harder. The comparative softness of the bulk material is due to the orientation of the basal planes, and the ease in which slip can occur along these planes. ${ }^{5,8}$ As seen in Fig. 2(a), such slip does not occur in the epilayers due to the strain compensation arising from the presence of threading dislocations. Thus the epitaxial layers are harder than their single crystal counterparts. Additionally, the presence of the sapphire substrate, may also further contribute to the comparative hardness of these layers. Work hardening of the epitaxial material was evident during the indentation process. Both the sapphire substrate, and the large damage accumulation beneath the indentation site are responsible for this. The large spread in data values, is likely a result of film inhomogeneity arising from the presence of varying densities of dislocations around the indentation site.

In conclusion, the mechanical properties of $\mathrm{ZnO}$ epitaxial layers grown on $a$ - and $c$-axis sapphire have been studied by nanoindentation and XTEM, and compared to the properties of single crystal material. It was found that unlike single crystal $\mathrm{ZnO}$, slip and the orientation of the basal planes did not play a key role in defining the properties of epilayer material. Instead, the underlying sapphire substrate governed the properties of the layers indirectly, by preventing damage propagation and slip, as well as introducing lattice mismatch which is responsible for the high dislocation density. Results of this study indicate that epilayer material is harder than its bulk counterpart, and experiences more localized contact induced deformation damage. This may have implications for the future design of epilayer based $\mathrm{ZnO}$ devices.

The authors wish to acknowledge Professor Mike Swain of the Biomaterials Science Research Unit, Department of Mechanical and Mechatronic Engineering and Faculty of Dentistry, The University of Sydney, for constructive comments and support. The work at UF is partially supported by the AFOSR under Grant Nos. F49620-03-1-0370 (T.S.) and NSF DMR 0400416. Dr. K. Koike of the Osaka Institute of Technology is acknowledged for help with the growth of $a$-axis epitaxial layers.

${ }^{1}$ D. C. Look, Mater. Sci. Eng., B 80, 383 (2001).

${ }^{2}$ J. E. Nause, III-V Rev. 12, 28 (1999).

${ }^{3}$ K. Ogata, K. Koike, T. Komuro, F. Yan, S. Sasa, M. Inoue, and M. Yano, J. Cryst. Growth 251, 623 (2003).

${ }^{4}$ Y. Chen, D. M. Bagnall, H. Koh, K. Park, K. Hiraga, Z. Zhu, and T. Yao, J. Appl. Phys. 84, 3912 (1998).

${ }^{5}$ S. O. Kucheyev, J. E. Bradby, J. S. Williams, C. Jagadish, and M. V. Swain, Appl. Phys. Lett. 80, 956 (2002).

${ }^{6}$ S. V. Prasad and J. S. Zabinski, Wear 203-204, 498 (1997).

${ }^{7}$ M. J. Mayo, R. W. Siegel, Y. X. Liao, and W. D. Nix, J. Mater. Res. 7, 973 (1992).

${ }^{8}$ J. E. Bradby, S. O. Kucheyev, J. S. Williams, C. Jagadish, M. V. Swain, P. Munroe, and M. R. Phillips, Appl. Phys. Lett. 80, 4537 (2002).

${ }^{9}$ M. J. Klopfstein, D. A. Lucca, and G. Cantwell, Phys. Status Solidi A 196, R1 (2003).

${ }^{10}$ J. Narayan, K. Dovidenko, A. K. Sharma, and S. Oktyabrsky, J. Appl. Phys. 84, 2597 (1998).

${ }^{11}$ T. Y. Tsui and G. M. Pharr, J. Mater. Res. 14, 292 (1999).

${ }^{12}$ J. S. Field and M. V. Swain, J. Mater. Res. 8, 297 (1993). 